\section{Maltrato en el pregrado de la carrera de Medicina: percepción de los estudiantes}

\author{
NANCY BASTÍAS-VEGA ${ }^{1, \mathrm{a}, \mathrm{h}}$, CRISTHIAN PÉREZ-VILLALOBOS ${ }^{1, \mathrm{~b}, \mathrm{i}}$, \\ DÉBORA ALVARADO-FIGUEROA ${ }^{1, \mathrm{c}}$, \\ MARY-JANE SCHILLING-NORMAN ${ }^{1, \mathrm{~d}}$, MARITZA ESPINOZA-RIFFO ${ }^{1, \mathrm{e}, \mathrm{h}}$, \\ PAULA PARRA-PONCE ${ }^{2, \mathrm{fh},}$, OLGA MATUS-BETANOCURT ${ }^{1, \mathrm{~g}, \mathrm{~h}}$, \\ JOSSELINNE TOIRKENS-NIKLITSCHEK ${ }^{2, \mathrm{~d}, \mathrm{i}}$
}

\section{Students' perception about mistreatment in undergraduate medical training}

Background: Alarms about mistreatment in medical education have been raised for almost 30 years. Aim: To describe the frequency of abuse reports among medical students at a university in Chile, investigating their association with age, sex, and educational level. Material and Methods: The Mistreatment by Teachers Questionnaire was applied to 264 first to seventh year medical students (54\% males). An exploratory factorial analysis of the instrument was performed, a descriptive analysis was made and its relationship with age, sex and level of training were evaluated. Results: Ninety eight percent of respondents reported having been mistreated at least once. Mistreatment was grouped into three factors with a confidence ranging between $\alpha=0.79$ and 0.93 , namely demoralization, deregulated demands and physical aggression. The first two were associated with age and level of education. There were no differences by sex. Conclusions: Mistreatment is common in undergraduate medical education, as it has been found in other universities around the world.

(Rev Med Chile 2021; 149: 617-625)

Key words: Bullying; Education, Medical, Undergraduate; Students, Medical; Teaching.
'Departamento de Educación

Médica, Universidad de

Concepción. Concepción, Chile.

2Dirección de Acompañamiento

Académico y Socioemocional,

Universidad Católica de Temuco.

Temuco, Chile.

aEnfermera.

bPsicólogo.

'Magíster en Educación Médica

para las Ciencias de la Salud.

dPsicóloga.

ePsicóloga y enfermera.

${ }^{\mathrm{f} K i n e s i o ́ l o g a . ~}$

Ingeniero en Informática.

hMagíster en Educación Médica

para las Ciencias de la Salud.

'Doctor en Ciencias de la

Educación.

'Magíster en Psicología m.

Psicología Educativa.

Trabajo financiado por el proyecto: Proyecto CONICYTFONDECYT Regular 1161541

Recibido el 13 de agosto de 2020, aceptado el 2 de noviembre de 2020 .

Correspondencia a:

Nancy Bastías-Vega

Chacabuco esquina Janequeo,

Departamento de Educación

Médica ( $5^{\circ}$ piso), Facultad

de Medicina, Universidad de Concepción. Concepción, Chile.

nbastias@udec.cl
(C

s posible que las escuelas de Medicina

$\checkmark$ sean un lugar donde sus alumnos son, de hecho, maltratados?". Eso se preguntaba Silver en 1982, mientras reflexionaba sobre las similitudes entre la experiencia de estos estudiantes de pregrado de Medicina (EPM) y los niños maltratados por sus cuidadores ${ }^{1}$.

Aunque la violencia en espacios educacionales lleva tiempo preocupando a la sociedad, se le ha prestado menos atención en educación superior ${ }^{2}$. Incluso, existen críticas de que su presencia se exagera. Empero, el maltrato en la formación médica no es un hecho aislado, sino que está extendido mundialmente $e^{3-5}$.
El maltrato incluye agresiones académicas como la privación de oportunidades, evaluaciones injustas; otras sociales, como la estigmatización, discriminación, humillación y crueldad psicológica y otras como el castigo físico, el acoso y la agresión sexual. Sin embargo, su definición es compleja ${ }^{6}$.

$\mathrm{Su}$ abordaje se ha realizado bajo diversos conceptos, como bullying ${ }^{7-13}$, maltrato ${ }^{3,15-17} \mathrm{o}$ acoso $^{18}$. El bullying se refiere al comportamiento hacia un individuo que es intimidante, degradante, ofensivo o malicioso, que daña su confianza y autoestima ${ }^{8}$, busca generar daño físico o psicológico y se sustenta en diferencias de poder o estatus entre agresor y víctima ${ }^{12}$. Y aunque parecería útil, 
autores como Olweus, lo definen como una forma de violencia entre pares ${ }^{14}$, lo que hace inadecuado aplicarlo a la educación médica, donde las fuentes más frecuentes de maltrato identificadas son los médicos especialistas o residentes de cursos superiores ${ }^{6,12,15}$. Esto ha hecho que se recurra a otros conceptos, como acoso y discriminación, definidos en el Acta de Igualdad de Reino Unido de 2010 como las conductas no deseadas que crean un ambiente intimidante, hostil, degradante, humillante $\mathrm{u}$ ofensivo y que afectan características protegidas como la etnia, religión, discapacidad, edad, género, orientación sexual o nivel socioeconómico $^{18}$.

Sin embargo, en este estudio, se ha optado por el concepto de maltrato, al constituir un concepto más amplio, que incluiría el carácter intencional de la conducta ${ }^{19}$, el daño físico y psicológico como su consecuencia ${ }^{12}$ y su origen en asimetrías de poder reales o simbólicas de la relación ${ }^{12,19}$.

\section{Frecuencia}

En la formación médica son frecuentes los estudios en residentes ${ }^{7,10,15,20}$. Sin embargo, la Medicina es una disciplina jerárquica. Los residentes están superditados a los médicos y los EPM están al final de la jerarquía ${ }^{8}$, haciendo a este último grupo de particular interés.

Ya en 1997, un estudio nacional en Estados Unidos de Norteamérica indicó que 96,5\% de los EPM reportaban a lo menos un incidente de maltrato en su formación, siendo las formas más frecuentes los comentarios despectivos y el menosprecio $^{3}$. Posteriormente, en un cuestionario aplicado nacionalmente se incorporó una pregunta al respecto a los graduados de ese país y se encontró que quienes reportaban haber sido maltratados personalmente fluctuaba entre 12 y $20 \%$ entre los años 2001 y 2011, siendo la humillación y el menoscabo las formas más frecuentes ${ }^{6}$.

En Europa, 63,3\% de los EPM de Reino Unido reportaban haber experimentado maltrato ${ }^{18} \mathrm{y}$ en Finlandia, un estudio realizado con estudiantes de los dos primeros años de distintas facultades mostró que el maltrato existía en todas ellas, con más de $40 \%$ de los estudiantes reportándose como víctimas. Aunque cada facultad tenía sus propios patrones de maltrato, el menosprecio, la humillación y los comentarios despectivos fueron más frecuentes en $\mathrm{EPM}^{16}$.

En Asia, el porcentaje de EPM que ha experi- mentado maltrato alguna vez fluctúa entre 28\% en Arabia Saudita ${ }^{9}, 52 \%$ en EPM de últimos años en Pakistán ${ }^{8}$ y $57 \%$ en EPM de segundo y tercer año en Jordania ${ }^{17}$.

En África, 100\% de los EPM de segundo a sexto año de Benín reportaba haber sido maltratado al menos una vez ${ }^{13}$.

En Latinoamérica, en Panamá, 18,2\% de los EPM de los primeros siete semestres declaraba haber sido víctima de maltrato ${ }^{11}$, y en Colombia, entre EPM de último año de Medicina, este porcentaje subía a $90,24 \%{ }^{12}$.

En Chile hay dos estudios en EPM: Uno en 2003, con alumnos de quinto año, y otro en 2006 con alumnos de segundo a séptimo año, donde los EPM que reportaban haber sido víctimas de maltrato eran $90,9 \%{ }^{21}$ y $91,7 \%{ }^{22}$, respectivamente. Posterior a ellos, solo hay un estudio en 2011 con residentes ${ }^{10}$.

Los resultados anteriores muestran una gran variabilidad, derivada de los distintos criterios de inclusión considerados, la diversidad de instrumentos y la falta de evidencias de su validez y confiabilidad. Sin embargo, esto no ha impedido que países como Estados Unidos de Norteamérica ${ }^{6}$ y Reino Unido ${ }^{18}$ reconozcan oficialmente el maltrato como un problema en la formación médica.

\section{Factores asociados}

En general, la aparición y extensión del maltrato se asocia a la naturaleza estresante, jerárquica y emocionalmente cargada del trabajo médico, a la deferencia de los EPM, a la institucionalización de algunas formas de discriminación y la subrepresentación de minorías ${ }^{17,18}$.

Pero el maltrato no es igual para todos. Se ha estudiado que el ser víctima o testigo de esto se asociaría a factores demográficos ${ }^{18}$, $\operatorname{como}$ el género. Así, las mujeres han mostrado ser más frecuentemente víctimas de maltrato entre funcionarios universitarios en España ${ }^{19}$, universitarios finlandeses ${ }^{16}$, residentes $^{15}$, y entre EPM de Estados Unidos de Norteamérica ${ }^{23}$ y Reino Unido ${ }^{18}$. Pero, en Pakistán y Jordania, el maltrato fue más frecuente en EPM hombres ${ }^{8,17}$ y en Colombia y Benín no se encontró diferencias por género ${ }^{12-13}$. Esto podría deberse a que el maltrato suele ser más frecuente para aquel género que es minoritario en cada escuela ${ }^{16}$.

Ser víctima también estaría asociado a otros factores, como al no ser caucásico ${ }^{7,21}$, la edad ${ }^{18,23}$ o a medir menos de $172 \mathrm{~cm}^{23}$. Asimismo, formas 
específicas de maltrato son más frecuentes en mujeres, personas más bajas, con IMC $>25^{9,18,22-23}$, no heterosexuales, con discapacidad o elementos relacionados a la religión ${ }^{18}$.

Además, en términos académicos, los alumnos en etapa de formación clínica tienden a experimentar más maltrato general ${ }^{18,22}$.

\section{Consecuencias}

El maltrato es un problema tanto a nivel organizacional como individual ${ }^{16}$.

A nivel individual, es percibido como una fuente de estrés en la formación ${ }^{15,16} \mathrm{y}$ afecta negativamente el bienestar emocional de los estudiantes ${ }^{4,24,25}$, pudiendo experimentar incluso síntomas de estrés postraumático ${ }^{25}$.

También afecta la relación con la carrera: Genera desconfianza en los sistemas de evaluación ${ }^{17}$, lleva a pensar en desertar de la carrera ${ }^{22}$ y un estudio de Sheehan ${ }^{26}$ mostró que un cuarto de los EPM habría elegido una carrera diferente si hubiesen sabido lo extendido que estaba el maltrato en la profesión. Adicionalmente, el maltrato impactaría negativamente las actitudes profesionales de los $\mathrm{EPM}^{4}$ al favorecer el cinismo y la erosión de los valores humanistas ${ }^{25}$.

Finalmente, afectaría la atención del paciente al perjudicar la comunicación y confianza en los equipos de salud $\mathrm{d}^{25}$.

Pese a lo anterior, las denuncias son poco frecuentes, ya que los EPM las consideran inaccesibles y poco efectivas, minimizan la situación o temen por las consecuencias académicas y sociales derivadas ${ }^{6,9,18}$.

\section{El problema}

Esta investigación busca describir la frecuencia con que los EPM de una universidad de Chile informan haber sido víctimas de maltrato, indagando su asociación con edad, género y nivel formativo. Se focaliza específicamente en el maltrato recibido desde los docentes, que serían la fuente más frecuente de este $e^{6,12,15}$. Adicionalmente, aporta evidencia psicométrica de la validez de constructo y confiabilidad de las mediciones del instrumento empleado.

\section{Método} versal.

\section{Participantes}

El estudio se realizó con EPM de una universidad tradicional chilena.

Mediante muestreo no probabilístico por cuotas, se encuestó a 264 EPM, de los cuales la mayoría eran hombres (53,8\%). Representaban desde primer a sexto año, ya que no se pudo acceder a los estudiantes de séptimo año (Tabla 1).

\section{Instrumentos}

Se empleó el Cuestionario de Maltrato desde Docentes, adaptado del cuestionario elaborado por Quine ${ }^{7}$, que ha sido usado en distintas versiones en Reino Unido ${ }^{7}$, Colombia ${ }^{12}$ y Chile $^{10}$. La adaptación empleada corresponde a la desarrollada por Bastías et al. ${ }^{10}$, aplicada previamente en residentes chilenos, pero sin estudios sobre su validez. Su adaptación consulta por el maltrato recibido específicamente desde los docentes, a diferencia de la versión de Quine, que consulta sobre maltrato en general. Presenta 20 afirmaciones sobre conductas de acoso o intimidación. Los EPM debían informar con qué frecuencia habían experimentado estas conductas durante su formación en el último semestre, empleando una escala de cinco alternativas (1: Nunca, 2: Casi nunca; 3: A veces, 4: Casi siempre y 5: Siempre).

\section{Procedimiento}

Se obtuvo la autorización del decano de la facultad participante como parte del proyecto FONDECYT Regular 1161541. Las encuestas fueron aplicadas en papel y presencialmente, previo proceso de consentimiento informado.

\section{Tabla 1. Caracterización de la muestra de estudiantes de Medicina}

\begin{tabular}{|lll|}
\hline Variable & & \\
Sexo & Hombre: & $142(53,8 \%)$ \\
& Mujer: & $122(46,2 \%)$ \\
Edad & M $=$ & 21,65 \\
& DE $=$ & 2,10 \\
& Mín $=$ & 18 \\
Nivel cursado & Máx $=$ & 33 \\
& Primer año: & $38(14,4 \%)$ \\
& Segundo año: $38(14,4 \%)$ \\
& Tercer año: & $40(15,2 \%)$ \\
& Cuarto año: & $46(17,4 \%)$ \\
& Quinto año: & $49(18,6 \%)$ \\
& Sexto año: & $53(20,1 \%)$ \\
\hline
\end{tabular}


El proyecto contó con la aprobación del Comité de Ética, Bioética y Bioseguridad de la Universidad de Concepción.

\section{Análisis}

El instrumento fue sometido a un análisis factorial exploratorio mediante método de eje principal (AEP), para identificar su estructura interna como evidencia de su validez de constructo, y se evaluó la confiabilidad de los factores empleando alfa de Cronbach ${ }^{27}$.

Luego se realizó un análisis de frecuencia de cada pregunta del cuestionario y de los factores identificados.

Sus puntajes fueron correlacionados con edad usando rho de Spearman, comparados por género usando U de Mann Whitney y comparados por nivel formativo usando Kruskal Wallis. Un valor de $\mathrm{p}<0,05$ se consideró estadísticamente significativo.

El análisis se realizó usando STATA SE 15.0.

\section{Resultados}

\section{Evaluación psicométrica}

Para obtener evidencia de validez de constructo de las mediciones del Cuestionario de Maltrato desde Docentes, se realizó un análisis factorial exploratorio, considerado adecuado, dado un estadístico $\mathrm{KMO}=0,91$ y una prueba Bartlett estadísticamente significativa, $\chi^{2}(190)=2977,91$; $p<0,001$.
Según el criterio de Kaiser-Guttman ${ }^{28}$, los 20 ítems se agrupaban en tres factores con autovalores mayores a $1,0(8,06,1,43$ y 1,05$)$. El mismo resultado arrojaron el gráfico de sedimentación (Figura 1) y el análisis paralelo de $\mathrm{Horn}^{27}$, que encontró tres factores con autovalores $(8,06$, $1,43$ y 1,05$)$ mayores a $95 \%$, observado en 1.000 muestras aleatorias calculadas $(0,65,0,55$ y 0,42$)$. Los tres factores explicaban $95,87 \%$ de la varianza total de los ítems.

La matriz de configuración, considerando rotación oblicua Oblimin (Tabla 2), mostró que todos los ítems presentaban, en algún factor, coeficientes superiores a 0,30 , que es la mínima carga significativa ${ }^{27}$, aunque el ítem 18 presentó cargas significativas en dos factores y fue asignado considerando aspectos teóricos y empíricos.

Los factores resultantes mostraron confiabilidades adecuadas ${ }^{29}$ (Tabla 3 ).

\section{Maltrato informado}

El 98,11\% ( $\mathrm{n}=259)$ de los EPM informó haber sido víctima de alguna forma de maltrato en el último semestre. Entre estas, la violencia física y hacia la propiedad personal fueron las menos frecuentes, informadas por $4,8 \%$ y $6,7 \%$ de los EPM, respectivamente. Solo un EPM informó la violencia hacia la propiedad como recurrente. Las formas de maltrato más frecuentes fueron la modificación inconsulta de responsabilidades y la subvaloración del desempeño, donde más de $10 \%$ de los casos dice que ocurre "siempre" o "casi siempre” (Tabla 4). En ninguno de los ítems se

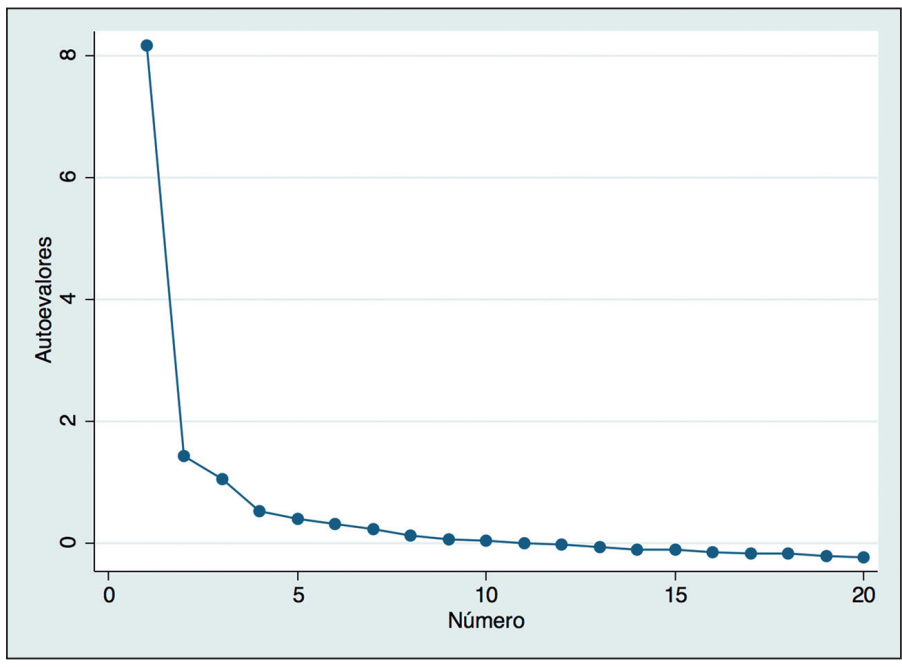

Figura 1. Gráfico de sedimentación del Cuestionario de Maltrato desde Docentes aplicado en estudiantes de Medicina. 
Tabla 2. Matriz de configuración del Cuestionario de Maltrato desde Docentes en estudiantes de medicina aplicando método de extracción de ejes principales y rotación oblicua Oblimin

\begin{tabular}{|cllcc|}
\hline $\mathbf{n}$ & Enunciado & I & II & III \\
\hline 1 & Intentos de menospreciar o menoscabar su trabajo & 0,722 & 0,032 & $-0,146$ \\
\hline 2 & Críticas injustificadas al supervisar/evaluar su quehacer & 0,773 & 0,102 & $-0,238$ \\
\hline 3 & Intentos de humillarle delante de sus pares & 0,849 & $-0,012$ & $-0,134$ \\
\hline 4 & Solicitud intimidatoria de realizar procedimientos de la disciplina & 0,765 & $-0,103$ & 0,116 \\
\hline 5 & Socavamiento de su integridad personal & 0,783 & $-0,114$ & 0,185 \\
\hline 6 & Insinuaciones dañinas o sarcásticas & 0,647 & 0,143 & 0,065 \\
\hline 7 & Amenazas verbales o no verbales & 0,562 & 0,108 & 0,252 \\
\hline 8 & Bromas inapropiadas acerca de usted & 0,631 & 0,069 & 0,141 \\
\hline 9 & Burlas persistentes & 0,634 & 0,029 & 0,266 \\
\hline 10 & Violencia física & $-0,024$ & 0,068 & 0,771 \\
\hline 11 & Violencia a la propiedad personal & 0,082 & 0,051 & 0,796 \\
\hline 12 & Le impiden acceder a información necesaria para sus estudios & 0,043 & 0,307 & 0,111 \\
\hline 13 & Le ignoran o le excluyen & 0,384 & 0,211 & 0,039 \\
\hline 14 & Negativas injustificadas a que pueda participar de otras actividades & 0,260 & 0,455 & 0,077 \\
\hline 15 & Exigencia exagerada en su quehacer & 0,259 & 0,540 & $-0,029$ \\
\hline 16 & Imposición de plazos imposibles de lograr & $-0,011$ & 0,513 & 0,132 \\
\hline 17 & Modificación de responsabilidades sin consultarle & $-0,039$ & 0,796 & 0,012 \\
18 & Subvaloración de su desempeño & 0,478 & 0,433 & $-0,124$ \\
\hline 19 & Intentos de desmoralizarle & 0,570 & 0,229 \\
\hline 20 & Cambios repentinos en las tareas asignadas sin consultarle & $-0,057$ & 0,716 & 0,088 \\
\hline
\end{tabular}

$\mathrm{N}=264$ (elaboración propia).

Tabla 3. Definición de los factores identificados, confiabilidad y estadísticos descriptivos del Cuestionario de Maltrato desde Docentes en estudiantes de Medicina

\begin{tabular}{|c|c|c|c|}
\hline Factor & $\mathbf{I}$ & II & III \\
\hline Nombre & Desmoralización & Exigencias desreguladas & Agresión física \\
\hline Definición & $\begin{array}{c}\text { Acciones que socavan la autoesti- } \\
\text { ma, seguridad y motivación de los } \\
\text { estudiantes }\end{array}$ & $\begin{array}{c}\text { Obstaculización del buen desem- } \\
\text { peño del estudiante a través de } \\
\text { exigencias desmedidas o carentes } \\
\text { de organización }\end{array}$ & $\begin{array}{c}\text { Ejercicio de la violencia hacia la } \\
\text { integridad del estudiante o sus } \\
\text { propiedades }\end{array}$ \\
\hline Ítems & $1,2,3,4,5,6,7,8,9,13,18$ y 19 & $12,14,15,16,17$ y 20 & 10 y 11 \\
\hline$\alpha$ & 0,93 & 0,79 & 0,86 \\
\hline M & 22,13 & 11,42 & 2,14 \\
\hline DE & 8,17 & 4,15 & 0,65 \\
\hline Mín & 12 & 6 & 2 \\
\hline Máx & 49 & 28 & 9 \\
\hline P25 & 16 & 8 & 2 \\
\hline P50 & 20 & 11 & 2 \\
\hline P75 & 27 & 14 & 2 \\
\hline Asimetría & 0,99 & 1,03 & 6,85 \\
\hline Curtosis & 3,69 & 4,50 & 59,60 \\
\hline
\end{tabular}

$N=264 ; \alpha$ : Alfa de Cronbach; M: Media aritmética; DE: Desviación estándar; Mín: Mínimo; Máx: Máximo; P: Percentil (elaboración propia). 
Tabla 4. Percepción de maltrato desde los docentes según los estudiantes de medicina

\begin{tabular}{|c|c|c|c|c|c|c|c|c|c|c|}
\hline & \multicolumn{2}{|c|}{ Nunca } & \multicolumn{2}{|c|}{ Rara vez } & \multicolumn{2}{|c|}{ A veces } & \multicolumn{2}{|c|}{$\begin{array}{l}\text { Casi } \\
\text { siempre }\end{array}$} & \multicolumn{2}{|c|}{ Siempre } \\
\hline & $\mathbf{n}$ & $\%$ & $\mathbf{n}$ & $\%$ & $\mathbf{n}$ & $\%$ & $\mathbf{n}$ & $\%$ & $\mathbf{n}$ & $\%$ \\
\hline Intentos de menospreciar o menoscabar su trabajo & 52 & 19,6 & 105 & 39,6 & 93 & 35,1 & 14 & 5,3 & 1 & 0,4 \\
\hline Críticas injustificadas al supervisar/evaluar su quehacer & 63 & 23,8 & 94 & 35,5 & 88 & 33,2 & 18 & 6,8 & 2 & 0,8 \\
\hline Intentos de humillarle delante de sus pares & 102 & 38,5 & 77 & 29,1 & 71 & 26,8 & 11 & 4,2 & 4 & 1,5 \\
\hline $\begin{array}{l}\text { Solicitud intimidatoria de realizar procedimientos de } \\
\text { la disciplina }\end{array}$ & 119 & 44,9 & 81 & 30,6 & 51 & 19,2 & 12 & 4,5 & 2 & 0,8 \\
\hline Socavamiento de su integridad personal & 152 & 57,4 & 79 & 29,8 & 27 & 10,2 & 7 & 2,6 & 0 & 0,0 \\
\hline Insinuaciones dañinas o sarcásticas & 110 & 41,5 & 77 & 29,1 & 61 & 23,0 & 14 & 5,3 & 3 & 1,1 \\
\hline Amenazas verbales o no verbales & 189 & 71,3 & 52 & 19,6 & 19 & 7,2 & 5 & 1,9 & 0 & 0,0 \\
\hline Bromas inapropiadas acerca de usted & 146 & 55,1 & 63 & 23,8 & 44 & 16,6 & 10 & 3,8 & 2 & 0,8 \\
\hline Burlas persistentes & 190 & 71,7 & 48 & 18,1 & 19 & 7,2 & 7 & 2,6 & 1 & 0,4 \\
\hline Violencia física & 255 & 96,2 & 6 & 2,3 & 3 & 1,1 & 1 & 0,4 & 0 & 0,0 \\
\hline Violencia a la propiedad personal & 250 & 94,3 & 11 & 4,2 & 3 & 1,1 & 0 & 0,0 & 1 & 0,4 \\
\hline $\begin{array}{l}\text { Le impiden acceder a información necesaria para sus } \\
\text { estudios }\end{array}$ & 190 & 71,7 & 40 & 15,1 & 31 & 11,7 & 3 & 1,1 & 1 & 0,4 \\
\hline Le ignoran o le excluyen & 147 & 55,7 & 51 & 19,3 & 48 & 18,2 & 16 & 6,1 & 2 & 0,8 \\
\hline $\begin{array}{l}\text { Negativas injustificadas a que pueda participar de } \\
\text { otras actividades }\end{array}$ & 174 & 65,7 & 48 & 18,1 & 31 & 11,7 & 8 & 3,0 & 4 & 1,5 \\
\hline Exigencia exagerada en su quehacer & 78 & 29,4 & 86 & 32,5 & 77 & 29,1 & 19 & 7,2 & 5 & 1,9 \\
\hline Imposición de plazos imposibles de lograr & 104 & 39,2 & 81 & 30,6 & 62 & 23,4 & 14 & 5,3 & 4 & 1,5 \\
\hline Modificación de responsabilidades sin consultarle & 98 & 37,0 & 64 & 24,2 & 66 & 24,9 & 29 & 10,9 & 8 & 3,0 \\
\hline Subvaloración de su desempeño & 98 & 37,0 & 67 & 25,3 & 69 & 26,0 & 23 & 8,7 & 8 & 3,0 \\
\hline Intentos de desmoralizarle & 145 & 54,7 & 66 & 24,9 & 41 & 15,5 & 9 & 3,4 & 4 & 1,5 \\
\hline $\begin{array}{l}\text { Cambios repentinos en las tareas asignadas sin } \\
\text { consultarle }\end{array}$ & 105 & 39,6 & 71 & 26,8 & 67 & 25,3 & 14 & 5,3 & 8 & 3,0 \\
\hline
\end{tabular}

$\mathrm{N}=264$ (elaboración propia).

identificó diferencias significativas entre hombres y mujeres.

Al correlacionar el maltrato informado con edad, se encontró que los alumnos mayores informaban desmoralización ( $r$ o $=0,36 ; \mathrm{p}<0,001$ ) $\mathrm{y}$ exigencias desreguladas $(\mathrm{rho}=0,13 ; \mathrm{p}<0,05)$ más frecuentes.

No se encontró diferencias estadísticamente significativas entre hombres y mujeres (Tabla 5). Sin embargo, sí se encontró diferencias por nivel formativo en desmoralización y exigencias desreguladas: la desmoralización fue percibida con mayor frecuencia en sexto y cuarto año, y con menor frecuencia en primer y segundo año. En tanto, las exigencias desreguladas ocurrirían más frecuentemente en sexto año.

\section{Discusión}

El 98,11\% de EPM informa haber sufrido maltrato, porcentaje superior al identificado en la mayoría de los estudios realizados en pregra$\mathrm{do}^{3,6,8-916-18,21-22}$, aunque cercano a $96,5 \%$ de estudiantes estadounidenses del estudio de Baldwin ${ }^{3}$, a 90,24\% en Colombia ${ }^{12}$, al 100\% en Benín ${ }^{13}$, y a los resultados previos en Chile que rondaban $90 \%{ }^{21-22}$.

Esto podría deberse a una creciente apertura 
Tabla 5. Comparación de la frecuencia de maltrato percibidos según sexo y nivel cursado en estudiantes de Medicina

\begin{tabular}{|c|c|c|c|c|}
\hline & & $\begin{array}{c}\text { Desmora- } \\
\text { lización }\end{array}$ & $\begin{array}{c}\text { Exigencias } \\
\text { desregu- } \\
\text { ladas }\end{array}$ & $\begin{array}{l}\text { Agresión } \\
\text { física }\end{array}$ \\
\hline \multicolumn{5}{|l|}{ Por sexo } \\
\hline \multirow[t]{4}{*}{ Hombres } & $M$ & 22,44 & 11,56 & 2,18 \\
\hline & $\mathrm{DE}$ & 8,62 & 4,61 & 0,78 \\
\hline & Md & 20,00 & 11,00 & 2,00 \\
\hline & $\mathrm{RI}$ & 12,00 & 6,00 & 0,00 \\
\hline \multirow[t]{4}{*}{ Mujeres } & M & 21,76 & 11,26 & 2,09 \\
\hline & DE & 7,63 & 3,55 & 0,46 \\
\hline & Md & 20,00 & 11,00 & 2,00 \\
\hline & $\mathrm{RI}$ & 10,00 & 6,00 & 0,00 \\
\hline \multicolumn{2}{|l|}{$\mathrm{p}$} & 0,73 & 0,87 & 0,26 \\
\hline \multicolumn{5}{|c|}{ Por nivel cursado } \\
\hline \multirow[t]{4}{*}{ Primero } & M & 18,18 & 11,05 & 2,03 \\
\hline & DE & 5,70 & 3,57 & 0,16 \\
\hline & Md & 16,00 & 11,00 & 2,00 \\
\hline & $\mathrm{RI}$ & 11,00 & 6,00 & 0,00 \\
\hline \multirow[t]{4}{*}{ Segundo } & M & 15,95 & 10,18 & 2,05 \\
\hline & DE & 3,65 & 3,57 & 0,32 \\
\hline & Md & 15,00 & 10,00 & 2,00 \\
\hline & $\mathrm{RI}$ & 5,00 & 6,00 & 0,00 \\
\hline \multirow[t]{4}{*}{ Tercero } & M & 22,55 & 10,85 & 2,10 \\
\hline & DE & 7,52 & 3,73 & 0,63 \\
\hline & Md & 20,50 & 11,00 & 2,00 \\
\hline & RI & 10,00 & 5,00 & 0,00 \\
\hline \multirow[t]{4}{*}{ Cuarto } & M & 24,96 & 11,22 & 2,35 \\
\hline & DE & 8,15 & 4,73 & 1,20 \\
\hline & Md & 23,50 & 10,00 & 2,00 \\
\hline & RI & 11,00 & 4,00 & 0,00 \\
\hline \multirow[t]{4}{*}{ Quinto } & M & 20,43 & 10,10 & 2,10 \\
\hline & $\mathrm{DE}$ & 6,89 & 3,19 & 0,42 \\
\hline & Md & 19,00 & 9,00 & 2,00 \\
\hline & RI & 9,00 & 4,00 & 0,00 \\
\hline \multirow[t]{4}{*}{ Sexto } & M & 28,17 & 13,66 & 2,15 \\
\hline & DE & 8,65 & 4,65 & 0,53 \\
\hline & $\mathrm{Md}$ & 27,00 & 13,00 & 2,00 \\
\hline & $\mathrm{RI}$ & 10,00 & 7,00 & 0,00 \\
\hline$p$ & & $<0,01 * * *$ & $<0,01 * * *$ & 0,11 \\
\hline
\end{tabular}

$\mathrm{N}=264 ;{ }^{*} \mathrm{p}<0,05 ;{ }^{*} \mathrm{p}<0,01 ;{ }^{* *} \mathrm{p}<0,001$; M: Media aritmética; DE: Desviación estándar; Md: Mediana; RI: Rango intercuartílico (elaboración propia). de los estudiantes a reconocer el maltrato en situaciones que antes normalizaban. No obstante, los informes superiores a $90 \%$ ya se encontraban en el estudio de Baldwin hace 23 años $^{3}$ y en los estudios chilenos en pregrado, que tienen más de 10 años $^{21-22}$.

Por otro lado, los estudios previos solían preguntar si el maltrato ha ocurrido alguna vez en la formación ${ }^{3,6,13}$, mientras que el presente estudio solo abordó el último semestre. Esto hace que, aunque el maltrato ocurriera "rara vez" según la mayoría de los EPM, su sola presencia implica una alerta académica.

El análisis factorial realizado organizó todas estas conductas en tres factores: desmoralización, exigencia desregulada y agresión física. Así, presenta una estructura conceptual que diferencia tres formas de agresión con propósito diferente que facilitarían su intervención y brindaría evidencia de la validez de constructo del instrumento empleado ${ }^{27}$.

La desmoralización es una de las formas de maltrato más frecuentemente identificadas por $\mathrm{EPM}^{6,8-9,16-18,22}$ y sus manifestaciones también fueron las más informadas en este estudio.

Otro factor alude a las exigencias desreguladas, que no implican "ser exigente", pues la exigencia motiva y favorece el aprendizaje. Implican realizar exigencias al estudiante que no son coherentes con el nivel cursado, los tiempos disponibles para realizar las tareas ni las oportunidades de aprendizaje otorgadas. Así, implica un error de planificación docente, donde las exigencias carecerían de validez curricular e instruccional ${ }^{27}$.

La violencia física aparece como tercer factor. Sus indicadores son los menos frecuentes: menos de $6 \%$ los ha vivido. Empero, su naturaleza hace que sea alarmante su mera aparición, pese a que otros estudios también lo identifican ${ }^{3,8-9,11,17}$.

En cuanto a las relaciones con otras variables, coherente con estudios previos, los EPM de mayor edad experimentan mayor desmoralización y exigencias desreguladas ${ }^{13,20}$. Esto puede estar asociado al nivel que cursan, pues los resultados muestran que los EPM de sexto año también sufren más estas formas de maltrato. Al respecto, la literatura indica que el maltrato se asocia directamente al avance académico ${ }^{9,11,17,22}$.

Broad destaca que es necesario que los equipos estén atentos a la aparición de microagresiones hacia grupos vulnerables, como las basadas en 
género ${ }^{18}$. En el presente estudio no aparecen diferencias entre hombres y mujeres. Pero el mayor maltrato a EPM de sexto año indicaría que son un grupo de riesgo que requerirían una atención similar, con una sensibilización especial de los involucrados en este nivel formativo.

En el mundo, la literatura ha transitado desde el diagnóstico del fenómeno a documentar iniciativas para eliminarlo ${ }^{5,25}$. Y aunque persisten las voces de docentes y estudiantes que buscan minimizarlo o atribuirlo a una mayor sensibilidad del alumnado, como si fuese un fenómeno 'millenial', no debemos olvidar que están pronto a cumplirse 30 años del artículo donde Silver denunciaba este fenómeno, ya de larga data.

Una limitación del estudio es que fue realizado en una sola universidad y mediante autorespuesta, pero sus resultados son coherentes con la literatura mundial, insinuando que no es un fenómeno aislado y que debe estudiarse en otras casas de estudio de Chile y Latinoamérica. Por otro lado, pese a la multiplicidad de estudios sobre el tema en Medicina, son pocos los que incluyen otras carreras ${ }^{16-17}$. Por lo que sería relevante diagnosticar, a lo menos, otros programas de pregrado en el área de salud.

Finalmente, es necesario reconocer la evidencia: el maltrato en Medicina está presente en todo el mundo, lleva años ahí1,3-5 y, lo más importante, no es una condición inherente a la formación. Al contrario, la socava ${ }^{4,25}$. El primer paso es dejar de negarlo.

\section{Referencia}

1. Silver HK. Medical students and medical school. JAMA 1982; 247 (3): 309-10.

2. Paredes OL, Sanabria-Ferrand PA, González-Quevedo LA, Moreno-Realphe SP. "Bullying" en las facultades de medicina colombianas, mito o realidad. Rev Fac Med 2010; 18 (2): 161-72.

3. Baldwin DC, Daugherty SR. Do residents also feel "abused"? Perceived mistreatment during internship. Acad Med. 1997; 72 (10 Suppl 1): S51-3.

4. Chung M, Thang C, Vermillion M, Fried J \& Uijtdehaage S. Exploring medical students' barriers to reporting mistreatment during clerkships: a qualitative study, Medical Education Online 2018; 23: 1, 1478170, DOI: 10.1080/10872981.2018.1478170.

5. Hasty N, Miller S, Merrell S, Lin D, Shipper E, Lau JN. Medical student perceptions of a mistreatment program during the surgery clerkship. Am J Surg. 2018; 215 (4): 761-6.

6. Mavis B, Sousa A, Lipscomb W, et al. Learning about medical student mistreatment from responses to the medical school graduation questionnaire. Acad Med. 2014; 89 (5): 705-11.

7. Quine L. Workplace bullying in junior doctors: questionnaire survey. BMJ 2002; 324: 878-9.

8. Ahmer S, Yousafzai AW, Bhutto N, Alam S, Sarangzai AK, et al. Bullying of Medical Students in Pakistan: A Cross-Sectional Questionnaire Survey. PLoS ONE 2008; 3 (12): e3889. doi:10.1371/journal.pone.0003889.

9. Alzahrani HA. Bullying among medical students in a Saudi medical school. BMC Res Notes 2012; 5 (1): 335. http://doi.org/q7d.

10. Bastías N, Fasce E, Ortiz L, Pérez C, Schaufele P. Bullying y acoso en la formación médica de postgrado. Rev Educ Cienc Salud 2011; 8 (1): 45-51.

11. Silva-Villarreal S, Castillo S, Eskildsen E, Vidal P, Mitre J, Quintero J. Prevalencia de bullying en estudiantes de los ciclos básicos y preclíni- cos de la carrera de medicina de la Universidad de Panamá. Arch Med 2013; 9 (4): 1-8.

12. Fernández N, Bonilla L, Rodríguez M, Sandoval G, Alzate JP, Murcia N, et al. Frequency of bullying perceived in clinical practices of last year interns of a medicine school: cross sectional study. Rev Fac Med. 2016; 64 (3): 447-52.

13. Akanni DWMM, Adjadohoun SBMG, Damien BG, et al. Maltraitance des étudiants et facteurs associés à la Faculté de Médecine de Parakou en 2018. Pan Afr Med J. 2019; 34: 150. doi:10.11604/pamj.2019.34.150.16367

14. Olweus D. Bullying at school: what we know and what we can do. Oxford: Wiley-Blackwell; 1993.

15. Daugherty SR, Baldwin DC, Rowley BD. Learning, satisfaction, and mistreatment during medical internship: a national survey of working conditions. JAMA 1998; 279 (15): 1194-9.

16. Rautio A, Sunnari V, Nuutinen M, Laitala M. Mistreatment of university students most common during medical studies. BMC Med Educ 2005; 5: 36. Published 2005 Oct 18. doi:10.1186/1472-6920-5-36.

17. Al-Hussain SM, Al-Haidari MS, Kouri NA, El-Migdadi F, Al-Safar RS, Mohammad MA: Prevalence of mistreatment and justice of grading system in five health related faculties in Jordan University of Science and Technology. Med Teach. 2008; 30 (3): e82-6.

18. Broad J, Matheson M, Verrall F, Taylor AK, Zahra D, Alldridge $\mathrm{K}$, et al. Discrimination, harassment and non-reporting in UK medical education. Med Ed 2018; 52 (4): 414-26. 
19. Justicia F, Benítez J, Fernández E. Caracterización del acoso psicológico en el contexto universitario. Revista de Psicología del Trabajo y de las Organizaciones 2006; 22 (3): 293-308.

20. Hoosen I, Callaghan R. A survey of workplace bullying of psychiatric trainees in the West Midlands. B J Psych Bulletin 2004; 28 (6): 225-7. doi: 10.1192/ pb.28.6.225.

21. Maida AM, Vásquez A, Herskovic V, Calderón JL, Jacard $\mathrm{M}$, Pereira A, et al. A report on student abuse during medical training. Med Teach 2003; 25: 497-501.

22. Maida AM, Herskovic V, Pereira A, Salinas-Fernández L, Esquivel C. Percepción de conductas abusivas en estudiantes de medicina. Rev Med Chile 2006; 134: 1516-23.

23. Chadaga AR, Villines D, Krikorian A. Bullying in the American Graduate Medical Education System: A National Cross-Sectional Survey. PLOS ONE 2016; 11 (3): e0150246. https://doi.org/10.1371/journal. pone. 0150246.

24. Heru A, Gagne G, Strong D. Medical student mistreatment results in symptoms of posttraumatic stress. Acad Psychiatry. 2009; 33 (4): 302-6.

25. Lind KT, Osborne CM, Badesch B, Blood A, Lowenstein SR. Ending student mistreatment: early successes and continuing challenges. Med Educ Online 2020; 25 (1): 1690846. doi:10.1080/10872981.2019.1690846.

26. Sheehan KH, Sheehan DV, White K, Leibowitz A, Baldwin DCJ (1990) A pilot study of medical student “abuse". JAMA; 263: 533-7.

27. Martínez R, Hernández MJ, Hernández MV. Psicometría. Madrid: Alianza; 2006.

28. Hair JF, Black WX, Babin BJ, Anderson RE, Tatham RL. Análisis multivariante. Madrid: Prentice Hall; 2005.

29. George D, Mallery P. SPSS for Windows step by step: A simple guide and reference. 11.0 update (4th ed.). Boston: Allyn \& Bacon; 2003. 